\title{
On the Hausdorff dimension of fractals given by certain expansions of real numbers
}

\author{
J. Neunhäuserer \\ Technical University Clausthal \\ Reitstallweg 9, D-38640 Goslar, Germany \\ neunchen@aol.com
}

\begin{abstract}
We transfer classical results on the Hausdorff dimension of $b$-adic and continued fraction expansions of real numbers to another expansion.
\end{abstract}

MSC 2010: 11K55, 28A80

Key-words: expansions of real numbers, Hausdorff dimension, fractals

\section{Introduction}

Dimension theory comes into the study of real numbers if we consider $b$-adic expansions

$$
\sum_{k=1}^{\infty} n_{k} b^{-k}
$$

where the digits $n_{k}$ comes from some non-empty subset $A \subseteq\{0,1, \ldots, b-1\}$ of all digits. The set $D(A)$ of such numbers is a fractal, its Hausdorff dimension is given by

$$
\operatorname{dim}_{H} D(A)=\frac{\log (\sharp A)}{\log b} .
$$

This result goes back to the work of Hausdorff [6]. If we prescribe the frequencies $\left(p_{0}, \ldots p_{b-1}\right) \in[0,1]^{b}$ of digits that appear in the expansion, we get a fractal $D\left(p_{0}, \ldots p_{b-1}\right)$ with Hausdorff dimension

$$
\operatorname{dim}_{H} D\left(p_{0}, \ldots p_{b-1}\right)=\frac{-\sum_{i=0}^{b-1} p_{i} \log \left(p_{i}\right)}{\log b} .
$$

This classical result is due to Besicovitch [1] for dyadic expansions and to Eggleston in the general form [2].

Now consider the continued fraction expansion of reals from $(0,1]$ in $\mathbb{N}^{\mathbb{N}}$,

$$
\frac{1}{n_{1}+\frac{1}{n_{2}+\frac{1}{n_{3}+\ldots}}}
$$

with prescribed digits $d_{k}$ coming from a finite set. We again get a fractal, and the Hausdorff dimension of this set was intensively studied since the work of Jarnik [7], who 
proved that the dimension of the set $E_{n}$ of continued fractions with digits in $\{1, \ldots, n\}$ can be estimated by

$$
1-\frac{4}{n \log 2} \leq \operatorname{dim}_{H} E_{n} \leq 1-\frac{1}{8 n \log n}
$$

for $n>8$. Nowadays we have an efficient non-trivial algorithm to calculate the dimension of $E_{n}$. In particular we have

$$
\operatorname{dim}_{H} E_{2}=0.5312805062772 \ldots
$$

see [8] a references there in.

In this paper we consider the expansion of the reals form $(0,1]$ in $\mathbb{N}^{\mathbb{N}}$ given by

$$
\sum_{k=1}^{\infty} 2^{-\left(n_{1}+n_{2}+\ldots+n_{k}\right)}
$$

and ask for the Hausdorff dimension of fractals related to this expansion. This expansion is induced by expanding Markov-Renyi maps, see [13].

In the next section we introduce our notations and prove results on the Hausdorff dimension of such expansion with prescribed digits. In the last section we state and prove our results for prescribed frequencies of the digits in such expansions.

\section{Results for prescribed digits}

Consider the set of infinite sequences of natural numbers,

$$
\mathbb{N}^{\mathbb{N}}=\left\{\left(n_{k}\right) \mid n_{k} \in \mathbb{N}\right\}
$$

and the map $\pi: \mathbb{N}^{\mathbb{N}} \longmapsto(0,1]$ given by

$$
\pi\left(n_{k}\right)=\sum_{k=1}^{\infty} 2^{-\left(n_{1}+n_{2}+\ldots+n_{k}\right)} .
$$

The sum converges since $2^{-\left(n_{1}+n_{2}+\ldots+n_{k}\right)}<2^{-k}$, and we have:

Proposition 2.1 $\Pi$ is a bijection.

Proof. Represent the numbers form $(0,1]$ using their binary expansions. With the convention that there is no tail of zeros in the expansion, this representation is unique. We have:

$$
\pi\left(n_{k}\right)=0 . \underbrace{0 \ldots 01}_{n_{1}} \underbrace{0 \ldots 01}_{n_{2}} \underbrace{0 \ldots 01}_{n_{3}} \cdots
$$

Now we see the $\pi$ is onto, i.e., invertible, since the representation on the right hand side can be arbitrarily prescribed. invertible, since the representation on the right hand side 
is unique.

So $\pi$ gives us a metric $d$ on $\mathbb{N}^{\mathbb{N}}$, via

$$
d\left(n_{k}, m_{k}\right)=\left|\pi\left(n_{k}\right)-\pi\left(m_{k}\right)\right|
$$

and we see that $\pi\left(n_{k}\right) \in \mathbb{Q}$ if and only if the sequence $\left(n_{k}\right)$ has periodic tails. Given a subset $A \subseteq \mathbb{N}$ we are interested in the set of numbers having a representation in $\mathbb{N}^{\mathbb{N}}$ with entries coming from $A$, this is

$$
\pi(A)=\Pi\left(A^{\mathbb{N}}\right)
$$

The set $\Pi(A)$ has a fractal geometry, thus we introduce Hausdorff dimension here. Recall $[4,12]$ that the $d$-dimensional Hausdorff measure of a set $A \subseteq \mathbb{R}$ is

$$
\mathfrak{H}^{d}(A)=\lim _{\epsilon \longmapsto 0} \inf \left\{\sum_{i=1}^{\infty} \operatorname{diam}\left(C_{i}\right)^{d} \mid A \subseteq \bigcup_{i=1}^{\infty} C_{i}, \operatorname{diam}\left(C_{i}\right)<\epsilon\right\} .
$$

The Hausdorff dimension of $A$ is given by

$$
\operatorname{dim}_{H} A=\sup \left\{d \mid \mathfrak{H}^{d}(A)=\infty\right\}=\inf \left\{d \mid \mathfrak{H}^{d}(A)=0\right\}
$$

Now we are able to state our main result on $\operatorname{dim}_{H} \Pi(A)$.

Theorem 2.1 For $A \subseteq \mathbb{N}$ let $d \in[0,1]$ be the solution of

$$
\sum_{n \in A} 2^{-n d}=1
$$

We have $\operatorname{dim}_{H} \Pi(A)=d$.

Proof. For $n \in A$ consider the contraction $T_{n}:(0,1] \longmapsto(0,1]$ given by

$$
T_{n} x=\left(\frac{1}{2}\right)^{n}(x+1)
$$

We have

$$
\begin{gathered}
\bigcup_{n_{0}=1}^{\infty} T_{n_{0}}(\Pi(A))=\bigcup_{n_{0}=1}^{\infty} T_{n_{0}}\left\{\sum_{k=1}^{\infty} 2^{-\left(n_{1}+n_{2}+\ldots+n_{k}\right)} \mid\left(n_{k}\right) \in A^{\mathbb{N}}\right\} \\
\left.=\bigcup_{n_{0}=1}^{\infty}\left\{\sum_{k=0}^{\infty} 2^{-\left(n_{0}+n_{1}+n_{2}+\ldots+n_{k}\right)}\right) \mid\left(n_{k}\right) \in A^{\mathbb{N}}\right\} \\
=\left\{\sum_{k=0}^{\infty} 2^{-\left(n_{0}+n_{1}+n_{2}+\ldots+n_{k}\right)} \mid\left(n_{k}\right) \in A^{\mathbb{N}}, n_{0} \in A\right\}=\Pi(A) .
\end{gathered}
$$

This means that $\Pi(A)$ is the attractor of the linear iterated function system $\left\{T_{n} \mid n \in A\right\}$ on $(0,1]$, see [6] for finite sets $A$ and [5] for infinite sets. 
This systems obviously fulfills the strong open set condition for the open interval $(0,1)$. This means $T_{m}((0,1)) \subseteq(0,1)$ with

$$
T_{m_{1}}((0,1)) \cap T_{m_{2}}((0,1))=\emptyset \text { for } m_{1} \neq m_{2}
$$

and $(0,1) \cap \Pi(A) \neq \emptyset$. The dimension formula in our result is the classical Moran formula. If $A$ is finite the result directly follows from the work of Moran [10]. If $A$ is infinite it follows from theory of infinite iterated function systems see theorem 3.11 of [5] or [11] for a more general approach.

Our main theorem has many interesting applications:

Corollary 2.1 For $n \geq 2$ we have $\operatorname{dim}_{H} \Pi(\{1, \ldots n\})=\log _{2}(s)$, where $s>1$ is the solution of

$$
s^{n}-s^{n-1}-\ldots-s=1 .
$$

In particular we have

$$
\operatorname{dim}_{H} \Pi(\{1,2\})=\log _{2}\left(\frac{\sqrt{5}+1}{2}\right)=0.6942419136 \ldots
$$

Proof. For $d>0$ we have

$$
\sum_{i=1}^{n} 2^{-i d}=\frac{2^{-d}-2^{-d(n+1)}}{1-2^{-d}}=1 \Leftrightarrow 2 \cdot 2^{-d}-2^{-d(n+1)}=1 \Leftrightarrow 2 \cdot 2^{d n}-2^{d(n+1)}=1 .
$$

Substituting $s=2^{d}$ gives $2 \cdot s^{n}-s^{n+1}=1$. Hence we get

$$
(s-1)\left(s^{n}-s^{n-1}-\ldots-s-1\right)=-2 s^{n}+s^{n+1}+1=0,
$$

leading to the result since $s \neq 1$. For $n=2$ we obtain $s^{2}-s-1=0$.

Corollary 2.2 Given $b \in \mathbb{N}$, let $A_{b}=\{(n b) \mid n \in \mathbb{N}\}$. We have $\operatorname{dim}_{H} \Pi\left(A_{b}\right)=1 / b$.

Proof. For $d>0$ we have

$$
\sum_{n=1}^{\infty} 2^{-n b d}=\frac{1}{1-2^{-b d}}-1=1 \Leftrightarrow d=1 / b
$$

Corollary 2.3 Given $b \geq 2$ and $c \in\{1, \ldots, b-1\}$, let $A_{b, c}=\left\{(n b+c) \mid n \in \mathbb{N}_{0}\right\}$. We have $\operatorname{dim}_{H} \Pi\left(A_{b, c}\right)=\log _{2}(1 / r)$ where $r \in(0,1)$ is the solution of

$$
r^{c}+r^{b}=1
$$

In particular we have

$$
\operatorname{dim}_{H} \Pi\left(A_{2,1}\right)=\operatorname{dim}_{H} \Pi(\{1,2\}) .
$$




\section{Proof.}

$$
\sum_{n=0}^{\infty} 2^{-(n b+c) d}=\frac{2^{-d c}}{1-2^{-b d}}=1 \Leftrightarrow 2^{-c d}+2^{-b d}=1
$$

Substituting $r=2^{-d}$ gives $r^{c}+r^{b}=1$ leading to the result. For $b=2$ and $c=1$ we have $r^{2}+r^{1}=1$. And if we set $s=1 / r, s$ fulfills $s^{2}-s-1=0$.

\section{Results for prescribed frequencies}

Let $\mathfrak{p}=\left(p_{i}\right)$ be a probability distribution on $\mathbb{N}$ which is supported by a finite set $A$, this means

$$
\sum_{i \in A} p_{i}=1 \quad \text { and } \quad p_{i}=0 \quad \text { for } \quad i \notin A .
$$

The expected value of $\mathfrak{p}$ is

$$
E(\mathfrak{p})=\sum_{i \in A} i p_{i}
$$

and the entropy is given by

$$
H(\mathfrak{p})=-\sum_{i \in A} p_{i} \log \left(p_{i}\right)
$$

We are interested in the set of numbers in $\Pi(A)$ with frequency $p_{i}$ of $i$ in the expansion $\pi^{-1}(x)$, this is

$$
A(\mathfrak{p})=\left\{\pi\left(n_{k}\right) \in \Pi(A) \mid \lim _{k \longmapsto \infty} \frac{\sharp\left\{l \mid n_{l}=i, \quad l=1, \ldots, k\right\}}{k}=p_{i}\right\} .
$$

Theorem 3.1 For a probability distribution $\mathfrak{p}$ on $\mathbb{N}$ which is supported by a finite set $A$ we have

$$
\operatorname{dim}_{H} A(\mathfrak{p})=\frac{H(\mathfrak{p})}{\log (2) E(\mathfrak{p})} .
$$

Proof. Consider the Borel probability measure $\mathfrak{m}$ on $\mathbb{N}^{\mathbb{N}}$ which is the infinite product of the probability measure given by $\mathfrak{p}$ on $\mathbb{N}$. Project this measure to $(0,1]$ using $\pi$

$$
\mu=\pi(\mathfrak{m})=\mathfrak{m} \circ \pi^{-1}
$$

By the law of large numbers we have

$$
\lim _{k \longmapsto \infty} \frac{\sharp\left\{l \mid n_{l}=i, \quad l=1, \ldots, k\right\}}{k}=p_{i} \quad \forall i \in A
$$

if we choose the digits of a sequence $\left(n_{k}\right)$ randomly from $A$ according to $\mathfrak{p}$. Hence this equation holds for $\mathfrak{m}$-almost all sequences in $\mathbb{N}^{\mathbb{N}}$. As a consequence we have $\mu(A(\mathfrak{p}))=1$. Now define intervals contained in $(0,1]$ by

$$
I_{n_{1} \ldots n_{k}}=T_{n_{k}} \circ \ldots \circ T_{n_{1}}((0,1])
$$


for $n_{i} \in A$ where the maps $T_{n}$ were defined in section 2. For $x \in \Pi(A)$ let $I_{n_{1} \ldots n_{k}}(x)$ be the unique interval containing $x$. Note that $\pi^{-1}(x)=\left(n_{1}, \ldots, n_{k}, \ldots\right)$. Furthermore let

$$
\sharp_{i}(x \mid k)=\sharp\left\{l \mid n_{l}=i, \quad l=1, \ldots, k\right\} .
$$

For the length of the intervals we obtain

$$
\log \left(\left|I_{n_{1} \ldots n_{k}}(x)\right|\right)=\log \left(2^{-\left(n_{1}+n_{2}+\ldots+n_{k}\right)}\right)=-\log (2) \sum_{i \in A} i \sharp_{i}(x \mid k)
$$

and for the measure of the intervals we get

$$
\log \mu\left(I_{n_{1} \ldots n_{k}}(x)\right)=\log \left(\prod_{j=1}^{k} p_{n_{j}}\right)=\sum_{i \in A} \sharp_{i}(x \mid k) \log p_{i}
$$

by the definition of $\mu$. If $x \in A(\mathfrak{p})$ we have

$$
\lim _{k \longmapsto \infty} \frac{\sharp_{i}(x \mid k)}{k}=p_{i}
$$

for all $i \in A$ by the definition of this set. Thus we have

$$
\begin{aligned}
\lim _{k \longmapsto \infty} \frac{1}{k} \log \frac{\mu\left(I_{n_{1} \ldots n_{k}}(x)\right)}{\left|I_{n_{1} \ldots n_{k}}(x)\right|^{s}} & =\lim _{k \longmapsto \infty} \frac{1}{k}\left(\sum_{i \in A} \sharp_{i}(x \mid k) \log p_{i}+s \log (2) \sum_{i \in A} i \sharp_{i}(x \mid k)\right) \\
& =-H(X)+s \log (2) E(X) .
\end{aligned}
$$

This implies for all $x \in A(\mathfrak{p})$

$$
\lim _{k \longmapsto \infty} \frac{\mu\left(I_{n_{1} \ldots n_{k}}(x)\right)}{\left|I_{n_{1} \ldots n_{k}}(x)\right|^{s}}=\left\{\begin{array}{cc}
0 & s<d \\
\infty & s>d
\end{array}\right.
$$

with

$$
d=\frac{H(\mathfrak{p})}{\log (2) E(\mathfrak{p})} .
$$

By the local mass distribution principle, see Proposition 4.9 of [4], we have $\mathfrak{H}^{s}(A(\mathfrak{p}))=\infty$ for $s<d$ and $\mathfrak{H}^{s}(A(\mathfrak{p}))=0$ for $s>d$. This implies $\operatorname{dim}_{H}(A(\mathfrak{p}))=d$.

Theorem 3.1 has following corollary.

Corollary 3.1 If $\mathfrak{p}$ has finite support, then $\operatorname{dim}_{H} A(\mathfrak{p})<1$.

Proof. The maximum of the dimension function $d(\mathfrak{p})=\operatorname{dim}_{H} A(\mathfrak{p})$ is given by the solution $d$ of

$$
\sum_{i \in A} 2^{-i d}=1
$$


for $p_{i}=2^{-i d}$. This solution is smaller than 1 .

On the other hand there exists a distribution on $\mathbb{N}$ which has full dimension. Namely, define the set of numbers in $\mathcal{A} \subseteq(0,1]$ with frequency $1 / 2^{i}$ of $i$ in the expansion $\pi^{-1}(x)$ by

$$
\mathcal{A}=\left\{\pi\left(n_{k}\right) \mid \lim _{k \longmapsto \infty} \frac{\sharp\left\{l \mid n_{l}=i, \quad l=1, \ldots, k\right\}}{k}=\frac{1}{2^{i}} \quad \forall i \in \mathbb{N}\right\} .
$$

Theorem 3.2 We have $\operatorname{dim}_{H} \mathcal{A}=1$.

Proof. Consider a Borel probability measure $\mathfrak{m}$ on $\mathbb{N}^{\mathbb{N}}$ which is the infinite product of an arbitrary probability distribution $\mathfrak{p}$ on $\mathbb{N}$. Project this measure to $(0,1]$ using $\pi$ to obtain a measure $\mu$ on $(0,1]$. Applying theorem A of [9] to the infinite iterated function system $\left\{T_{n} \mid n \in \mathbb{N}\right\}$ we obtain that the Hausdorff dimension of $\mu$,

$$
\operatorname{dim}_{H}(\mu)=\inf \left\{\operatorname{dim}_{H} A \mid \mu(A)=1\right\}
$$

is given by

$$
\operatorname{dim}_{H} \mu=\frac{\sum_{i=1}^{\infty} p_{i} \log \left(p_{i}\right)}{\sum_{i=1}^{\infty} p_{i} \log \left(2^{-i}\right)} .
$$

Now inserting $p_{i}=1 / 2^{i}$ we get $\operatorname{dim}_{H} \mu=1$. But by the law of large numbers $\mu(\mathcal{A})=1$. Hence $\operatorname{dim}_{H} \mathcal{A} \geq 1$, which obviously implies the result.

To prove an upper bound on $\operatorname{dim}_{H} A(\mathfrak{p})$ for arbitrary probability sequences $\mathfrak{p}$ seems to be a hard problem, the proof of Theorem 3.1 does not work. In probabilistic terminology, if we have a sequence of unbounded random variables, the expected value and entropy do not have to converge to the expected value and entropy of the limit.

\section{References}

[1] A.S. Besicovitch, On the sum of digits of real numbers represented in the dyadic system, Math. Ann. 110, 321-330, 1934.

[2] H.G. Eggleston, The fractional dimension of a set defined by decimal properties, Quart. J. Math. Oxford Ser. 20, 6-31, 1949.

[3] R. J. Evans, J. Boersma, N. M. Blachman and A. A. Jagers, The Entropy of a Poisson Distribution: Problem 87-6, SIAM Rev. Vol. 30, no. 2, 314-317, 1988.

[4] K. Falconer, Fractal Geometry - Mathematical Foundations and Applications, Wiley, New York, 1990. 
[5] H. Fernau, Infinite iterated function systems, Math. Nach., vol. 170, issue 1, 79-91, 1994.

[6] J.E. Hutchinson, Fractals and self-similarity, Indiana Univ. Math. J. 30, 271-280, 1981.

[7] V. Jarnik, Zur metrischen Theorie der diophantischen Approximationen, Prace Mat.-. Fix. 36, 91-106, 1928.

[8] O. Jenkinson and M. Pollicott, Computing the dimension of dynamically defined sets: E2 and bounded continued fractions, Erg. Th. and Dyn. Sys., 21, 1429-1445, 2001.

[9] M. Moran and J.-M. Rey, Geometry of self-similar measures, Ann. Aca. Scien. Fennic Math., vol. 22, 365-386, 1997.

[10] P. Moran, Additive functions of intervals and Hausdorff measure, Proc. Camb. Phil. Soc. 42, 15-23, 1946.

[11] R.D. Mauldin and M. Urbanski, Dimensions and measures in infinite iterated function systems, Proc. London Math. Soc., 73, 105-154, 1996.

[12] Ya. Pesin,Dimension theory in dynamical systems: contemporary view and applications, The University of Chicago Press 1997.

[13] M. Pollicott and H. Weiss, Multifractal analysis of Lyapunov exponent for continued fraction and MannevillePomeau transformations and applications to diophantine approximation, Comm. Math. Phy., 207, 145-171, 1999. 